Pathologe 2010 · [Suppl 2] 31:263-267

DOI 10.1007/s00292-010-1320-3

Online publiziert: 15. August 2010

(c) Springer-Verlag 2010

\author{
K.-F. Becker ${ }^{1} \cdot$ D. Berg ${ }^{1} \cdot$ K. Malinowsky ${ }^{1} \cdot$ C. Wolff ${ }^{1}$ - B. Ergin ${ }^{1} \cdot$ S. Meding ${ }^{2} \cdot$ A. Walch ${ }^{2}$. \\ H. Höfler ${ }^{1,2}$ \\ ${ }^{1}$ Institut für Pathologie, Technische Universität München \\ ${ }^{2}$ Institut für Pathologie, Helmholtz Zentrum München, \\ Deutsches Forschungs-zentrum für Gesundheit und Umwelt, München
}

\section{Neues zur Proteinanalytik archivierter Gewebeproben}

gie und Integrität der Makromoleküle, sie spielen aber in der weltweiten Krankenhausroutine derzeit keine entscheidende Rolle.

\section{Schnittbasierte Proteinanalysen von FFPE-Geweben}

Während Nukleinsäure-basierte Analysen von FFPE-Gewebeproben schon länger erfolgreich angewendet werden, stehen Untersuchungen auf Proteinebene erst am Anfang (mit Ausnahme der Immunhistochemie). Es zeichnet sich jedoch ab, dass viele Proteinuntersuchungsmethoden, die an frischen oder gefrorenen Ge- webeproben eingesetzt werden, auch an nen. Prinzipiell können die Proteinanalysen klinischer Gewebeproben in schnittbasierte und extraktbasierte Methoden unterschieden werden (• Abb. 1).

Ausgehend vom FFPE-Gewebeblock gehören zu den schnittbasierten Methoden die Immunhistochemie, auf die hier nicht näher eingegangen wird, und die „matrix-assisted laser desorption/ionization“- (MALDI-) Massenspektrometrie. Mit ihren vielfältigen Verfahren und Anwendungsfeldern stellt die Grundtechnik der Massenspektrometrie eine wesentliche Schlüsseltechnologie der biomediziFFPE-Proben angewendet werden kön-

Seit Jahrzehnten werden weltweit FFPEGewebeproben routinemäßig zur histopathologischen Krankheitsdiagnose verwendet. Der landläufigen Meinung internationaler Forscher zufolge sind jedoch FFPEGewebeproben für quantitative Proteomanalysen nicht geeignet $[2,10,13]$. Alternativen zur Formalinfixierung sind z. B. das Einfrieren der Gewebe und die aktuell diskutierten experimentellen Fixiermethoden mit z. B. 70\%igem Ethanol [2, 13]. FFPE-Gewebe bietet den Vorteil, dass die Gewebemorphologie sehr gut erhalten bleibt. Proteine konnten allerdings lange Zeit in diesen fixierten Geweben nicht adäquat untersucht werden. Gefriermaterial ist zwar eine exzellente Quelle für die Extraktion von Makromolekülen, die Sammlung, Prozessierung und Lagerung der Gewebe ist jedoch sehr aufwendig und kostenintensiv. Die experimentellen Fixiermethoden sind zwar ein guter Kompromiss zwischen erhaltener Morpholo-

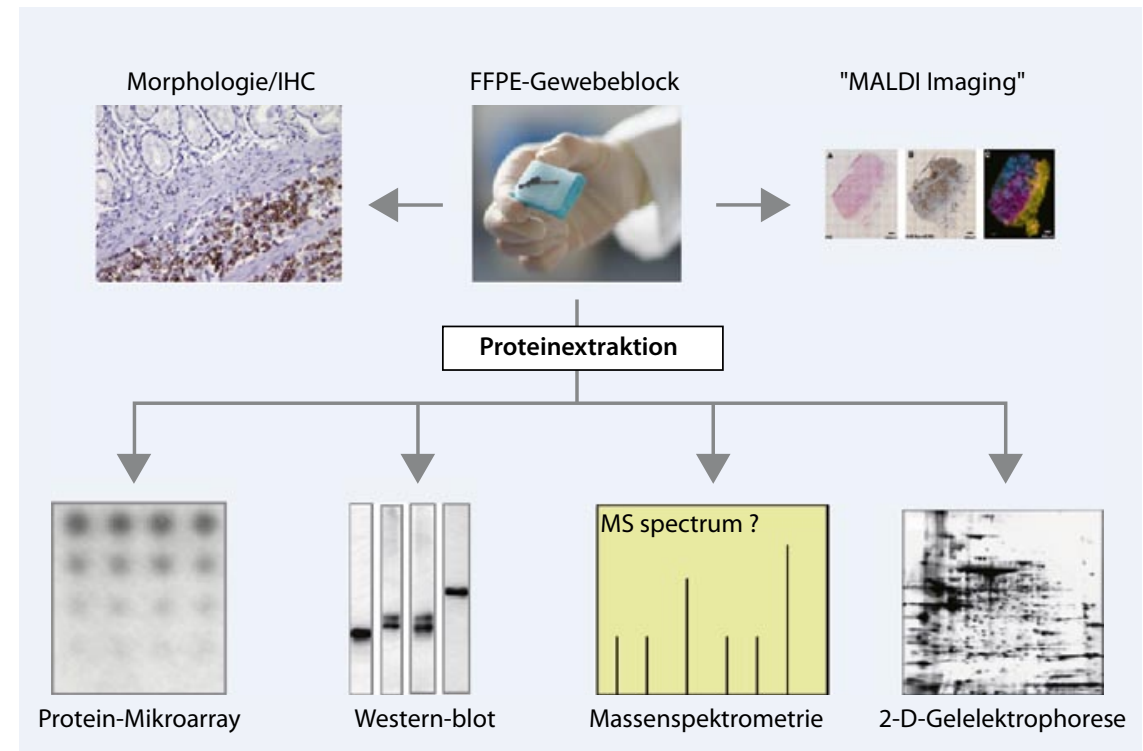

Abb. 1 A Proteomanalyse klinischer Gewebeproben. Durch Ergebnisse der letzten Jahre zeichnet sich $\mathrm{ab}$, dass viele Proteinuntersuchungsmethoden, die an frischen oder gefrorenen Gewebeproben eingesetzt werden, auch an FFPE-Proben angewendet werden können. Prinzipiell können die Proteinanalysen klinischer Gewebeproben in schnittbasierte (oben) und extraktbasierte (unten) Methoden unterschieden werden 
Tab. 1 Bildgebende Massenspektrometrie an FFPE-Geweben

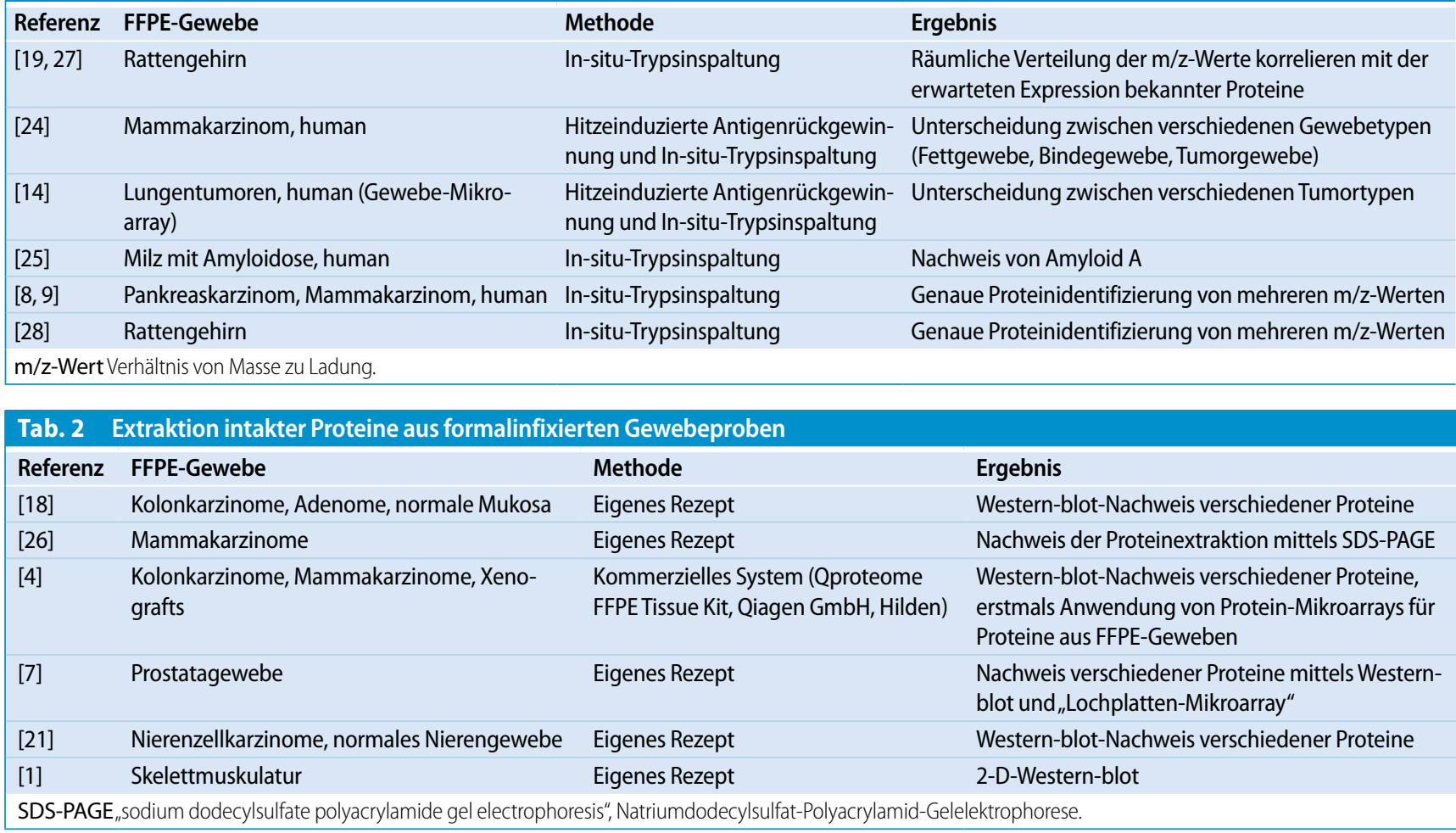

nischen Forschung und in zunehmendem Umfang in der Analyse von Geweben dar. Durch eine neue Entwicklung, die Massenspektrometrie und mikroskopische Bildgebung verbindet („MALDI Imaging"), können Proteine direkt im Gewebe sichtbar gemacht werden, wodurch sich neue Möglichkeiten für die Zelltypisierung und die Erforschung von Arzneistoffen ergeben. Die Korrelation der klassischen Histologie mit molekularen bildgebenden Verfahren führt somit zu einer neuen Qualität von Daten für die medizinische Diagnostik, die es erlaubt, molekulare Ursachen von Krankheiten besser zu verstehen [31]. Kürzlich sind mehrere Artikel erschienen, die zeigten, dass prinzipiell die Methode der MALDIImaging-Massenspektrometrie auch an FFPE-Proben angewendet werden kann (• Tab. 1).

\section{Extraktbasierte Proteinanalysen von FFPE-Geweben}

$\mathrm{Zu}$ den extraktbasierten Methoden gehören z. B. Western-blot, Protein-Mikroarray und 2-D-Gelelektrophorese. Entgegen den langjährigen Überzeugungen in der Wissenschaftsgemeinde $[2,10,13]$ konn- te in unserer Arbeitsgruppe - und in anderen Labors - eine Methode entwickelt werden, die es ermöglicht, intakte und immunreaktive Proteine aus FFPE-Gewebeproben in Lösung zu bringen ( $\bullet$ Tab. 2). Zudem lassen sich die Proteine so aus den Gewebeproben extrahieren, dass erstmalig eine exakte Bestimmung der Expressionsstärke möglich wird. Die isolierten Proteine können dann nachgeordnet mit etablierten Nachweismethoden, wie z. B. Western-blot, weiter untersucht werden. Histologische Färbungen stören jedoch die Proteinextraktion. Wir empfehlen daher, die so genannte ,kontrollierte Proteinextraktion" anzuwenden, die von Hämatoxylin-Eosin- (HE-) gefärbten Referenzschnitten und nachfolgenden ungefärbten Schnitten ausgeht [5].

\section{Protein-Mikroarrays}

Um dynamische Prozesse in Tumorzellen und die komplexen Vernetzungen ihrer intrazellulären Signalketten darstellen zu können, wurde die Technik der Reverse-Phase-Proteinarrays (auch Proteinlysat-Microarray genannt) weiterentwickelt $[3,12,22]$. Typischerweise werden bei einem Proteinlysat-Mikroarray Na- nolitermengen von Zell- oder Gewebelysaten mit einem Roboter auf eine Oberfläche (z. B. Nitrozellulose) aufgebracht. Anschließend wird das Zielprotein mit einem spezifischen Antikörper direkt oder indirekt detektiert (- Abb. 2). Die entscheidenden Vorteile dieser Technik gegenüber anderen Methoden (z. B. Westernblot) sind:

- sehr wenig Materialverbrauch (im Nanoliterbereich),

- Verdünnungskurven, linearer Bereich der Antikörper-Antigen-Reaktion,

- Replikate,

- Hochdurchsatzverfahren,

- Analyse vieler Proben unter exakt den gleichen Bedingungen möglich,

- kompatibel mit gängigen immunchemischen Verfahren, einschließlich Signalverstärkung,

- Automatisierung möglich (kompatibel zu modernen vollautomatischen Färbesystemen für die Immunhistochemie).

Die Proben werden in Verdünnungsreihen und Replikaten auf einen Nitrozellulose-beschichteten Objektträger aufgebracht. Damit wird sichergestellt, dass die Antikörper-Antigen-Reaktion im- 
mer im linearen Bereich gemessen wird. Auf einem Array können Proben vieler Tumorpatienten oder zu unterschiedlichen Zeitpunkten isolierte Proben eines Patienten untersucht und mit Referenzlysaten verglichen werden. Somit kann beispielsweise das Signalkaskadenprofil des Tumors eines Patienten vor, während und nach Anwendung zielgerichteter Therapien untersucht werden, sodass die Wirkung der Therapien oder sich entwickelnde Resistenzen auf der Ebene der Signalproteine dargestellt werden können. Die Sensitivität der Methode erlaubt den Nachweis selbst ausgesprochen schwacher Signale, wie im Falle von phosphorylierten Transkriptionsfaktoren. Gerade in Biopsien, bei denen probenbedingt wenig Material zur Verfügung steht, können auf diese Weise hunderte von Proteinen quantitativ bestimmt werden. Auf diese Art ist es möglich, ein individuelles Signalprofil jedes Patienten im klinischen Routineprozess zu erstellen, was in Zukunft eine optimale Patienten- und Therapieselektion gewährleisten kann $[15,20,23,29,32]$.

Ein großer limitierender Faktor für eine erfolgreiche Anwendung der ProteinMikroarray-Technik besteht in der Verfügbarkeit von spezifischen Antikörpern. Bei unseren eigenen Analysen werden nur Antikörper im Protein-Array verwendet, die im Western-blot von FFPE-Lysaten ein klares Ergebnis liefern: nämlich eine spezifische Bande beim erwarteten Molekulargewicht des untersuchten Proteins.

Eine andere Herausforderung bei der Array-Analyse sind die Variabilität und Vergleichbarkeit der Signalintensitäten zwischen verschiedenen Arrays, die mit demselben Antikörper detektiert wurden. Durch Referenzstandards, die zusammen mit den Patientenproben auf jeden Array aufgebracht werden, lassen sich die Gesamtproteinmenge und die Intensität der Proteine zwischen verschiedenen Arrays hervorragend vergleichen [6].

\section{Präanalytische Parameter}

Es ist heute unbestritten, dass biologische Proben immer mehr zur Schlüsselressource für die erfolgreiche biomedizinische Forschung und Medikamentenentwicklung werden. Solche Proben werden typischerweise in Biobanken verwaltet, d. h.

Pathologe 2010 · [Suppl 2] 31:263-267 DOI 10.1007/s00292-010-1320-3

(c) Springer-Verlag 2010

\section{K.-F. Becker · D. Berg · K. Malinowsky · C. Wolff · B. Ergin · S. Meding · A. Walch · H. Höfler Neues zur Proteinanalytik archivierter Gewebeproben}

\section{Zusammenfassung}

Gewebeproben werden seit Jahrzehnten weltweit routinemäßig zur histopathologischen Charakterisierung verwendet, um erkranktes von gesundem Gewebe zu unterscheiden. Während Nukleinsäure-basierte Analysen formalinfixierter und paraffineingebetteter (FFPE-) Gewebeproben schon länger erfolgreich angewendet werden, stehen Untersuchungen auf Proteinebene erst am Anfang (abgesehen von der Immunhistochemie). Es zeichnet sich jedoch ab, dass viele Proteinuntersuchungsmethoden, die an frischen oder gefrorenen Gewebeproben eingesetzt werden, auch an FFPE-Proben angewendet werden können. Hierzu gehören z. B. Western-blot, Protein-Mikroarray, bildgebende Massenspektrometrie („MALDI Ima- ging") und 2-D-Gelelektrophorese. Diese Ergebnisse sind überraschend, da die Wissenschaftsgemeinde lange der Überzeugung war, dass FFPE-Proben für Proteinanalysen außer Immunhistochemie - nicht geeignet sind. In diesem Übersichtsbeitrag berichten wir über neueste Entwicklungen auf diesem Gebiet und gehen dabei besonders auf quantitative Proteinbestimmungen und Hochdurchsatztechniken ein, die in Zukunft in den Routineablauf zur Proteinbiomarkerbestimmung integriert werden können.

\section{Schlüsselwörter}

Proteomics · FFPE - Protein-Microarray ·

Molekulare Diagnostik. Gewebe

\section{Update on protein analysis of fixed tissues}

\section{Abstract}

Tissue samples have been routinely used for decades to distinguish healthy from diseased tissue in histopathological characterization. While nucleic acid-based methodologies have been successfully in use for many years, protein-based techniques, in contrast, are at a very early stage (with the exception of immunohistochemistry). One reason for this delay may be that the scientific community has long thought that formalin-fixed and paraffin embedded (FFPE) tissues are unfit for protein analysis. However, recent reports demonstrate that many protein methods that are routinely used for frozen tissues can also be applied for FFPE tissues, including Western blot, protein microarray, matrix-assisted laser desorption/ionization (MALDI) imaging and $2 \mathrm{D}$ gel electrophoresis. The present article provides an overview of recent developments in this field, focussing particular attention on quantitative analysis and high throughput technologies that have the potential to be integrated into the routine workflow of clinical pathology laboratories.

\section{Keywords}

Proteomics · FFPE · Protein microarray · Molecular diagnostics - Tissue 


\section{Hauptreferate: Molekulare Tumorpathologie}

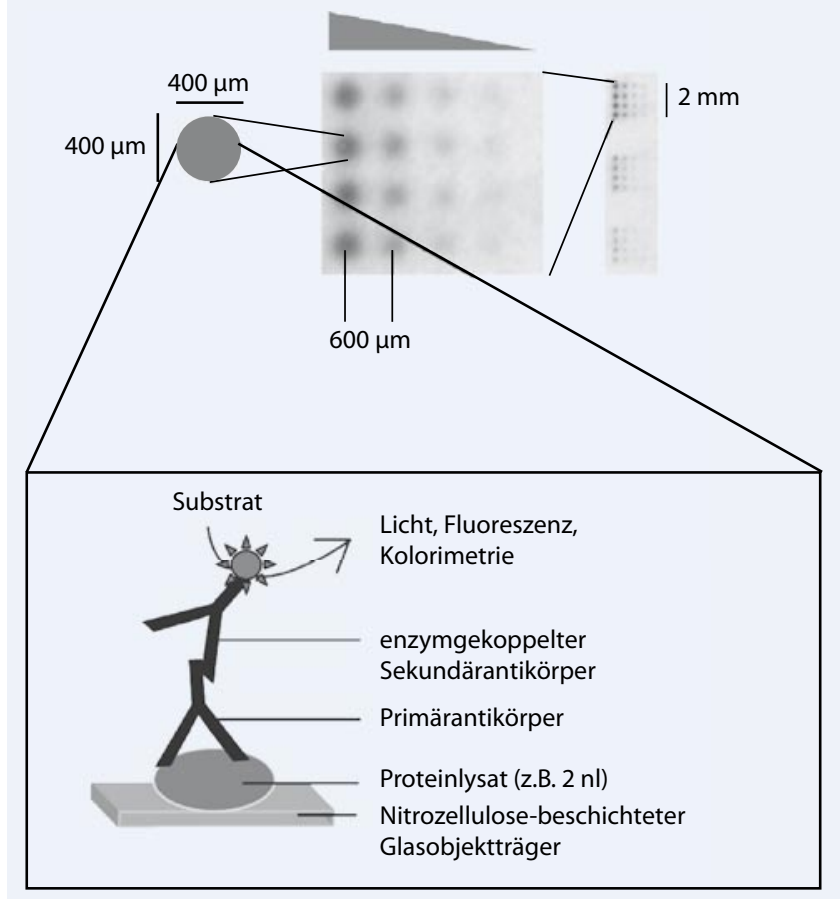

Abb. $2 \Delta$ Bei der Protein-Mikroarray-Technik werden Nanolitermengen von Proteinlysaten auf einem mit Nitrozellulose beschichteten Glasobjektträger immobilisiert und die Zielproteine mit spezifischen Antikörpern detektiert. Da Verdünnungsreihen auf die Oberfläche aufgebracht werden, ist gewährleistet, dass die Antikörper-Antigen-Reaktion im linearen Bereich bestimmt wird

in Einrichtungen, die biologische Proben sammeln, prozessieren, lagern und annotieren. Eine besondere Herausforderung für die Untersuchung klinischer Gewebeproben ist die Standardisierung der Probengewinnung, da Einflüsse von Medikamenten, Operationsverfahren und Erfordernisse der pathologischen Diagnostik durch die individuellen Bedürfnisse der Patienten bestimmt sind. Es sind systematische Studien erforderlich, um zu evaluieren, wie sich die verschiedenen Parameter (z. B. perioperative Medikation, Ischämiezeiten, Gewebeprobenprozessierung, -konservierung und -lagerung) auf die Probenqualität auswirken [30]. Die diagnostischen Marker sind möglicherweise instabil und könnten sich während der Zeit zwischen Entnahme der Probe und letztendlicher Analyse verändern, was zu einer Verfälschung der Ergebnisse führen kann.

Trotz der weltweiten Bedeutung der molekularen Diagnostik gibt es bisher noch keine standardisierten Abläufe für diese entscheidende präanalytische Phase [17]. Die USA und Europa haben auf den zunehmenden Bedarf an biologischen
Proben, besonders Gewebeproben, reagiert. Viele der älteren Proben entsprechen hinsichtlich der Annotation klinischer Daten und/oder der Gewebeprobenqualität nicht mehr heutigen Ansprüchen. In den USA wurde vom „National Cancer Institute" (NCI) im Jahre 2005 eigens das „Office of Biorepositories and Biospecimen Research“ (OBBR) gegründet. Aufgabe des OBBR ist sicherzustellen, dass menschliche Gewebeproben mit der höchsten Qualität für biomedizinische Forschungen zur Verfügung stehen. Auch die EU hat auf die steigenden Bedürfnisse für die Gewebeprobenqualität im Bereich der molekularen Diagnostik reagiert: ein EU-Konsortium mit dem Namen SPIDIA („Standardisation and improvement of generic Pre-analytical tools and procedures for In-vitro DIAgnostics"), hat sich zum Ziel gesetzt, Regeln für die Probenvorbereitung diagnostischer Biomarkertests zu erarbeiten (http://www.spidia.eu). Eine paneuropäische Infrastruktur, die „Biobanking and Biomolecular Resources Research Infrastructure“ (BBMRI), hat ein Konzept entwickelt, wie Biobanken vernetzt werden und somit in ihrer Nutzung eine Effektivitätssteigerung erzielen können.

Welche Parameter beeinflussen die Qualität der Gewebeprobe am stärksten? Wichtige Aspekte der präanalytischen Phase sind die Gewebeentnahme, der Transport, die Temperatur, die Fixierung des Gewebes sowie die Lagerung der Proben (• Abb.3). Alle diese Schritte können Einfluss auf die Expression der Krankheitsmarker haben. Trotz der weltweiten Bedeutung der molekularen Diagnostik gibt es bisher erst wenige systematische Untersuchungen für diese entscheidende präanalytische Phase. Erste Studien mit Gefriermaterial konnten zeigen, dass Verzögerungen zwischen Gewebeentnahme und Einfrierung der Proben die Expression von Proteinen verändern kann. Das Gewebe ist weiterhin „lebendig“, und die Qualität wie auch Quantität von molekularen Markern, wie z. B. das Phosphoproteom, verändern sich [11].

Durch die Fortschritte bei der Proteinuntersuchung von FFPE-Proben können jetzt auch formalinfixierte Gewebeproben im klinischen Routineprozess hinsichtlich Integrität, Stabilität und Expression von Proteinbiomarkern untersucht werden. Das war in dieser Form bislang noch nicht möglich; deswegen gibt es keinerlei Daten in der Literatur zum Einfluss der Gewebebehandlung und -fixierung auf die Expression bzw. Deregulation von 
Proteinen in fixierten Gewebeproben. Eine genaue Dokumentation der Gewebeprozessierung, die bereits bei der Gefäßligations- und Resektatentnahmezeit beginnt, wird in Zukunft nötig werden. Außerdem sollte die Medikation des Patienten bei jeder Probe erfasst werden. Die Fortschritte bei der Proteinuntersuchung formalinfixierter Gewebeproben erlauben nun erstmals, den Einfluss der präanalytischen Phase im klinischen Routineprozess genauer zu studieren.

\section{Ausblick}

Neben Nukleinsäure-basierten Analysen und der Immunhistochemie werden extraktbasierte Proteinuntersuchungen für Diagnose und Therapie immer bedeutsamer. Diese Untersuchungen werden nur nachhaltig zum Erfolg führen, wenn die entdeckten Marker der spezifischen Krankheit zugeordnet werden können und nicht durch die Gewebeprozessierung entstehen. Unsere Arbeitsgruppe ist derzeit dabei, Proteinbiomarker für die Gewebeprobenqualität zu entwickeln. Solche Marker könnten - extraktbasiert - am FFPE-Material zusammen mit den Krankheitsmarkern mittels Hochdurchsatztechniken wie den Protein-Mikroarrays quantitativ bestimmt werden und $\mathrm{zu}$ verlässlicheren Ergebnissen der molekularen In-vitro-Diagnostik führen.

\section{Korrespondenzadresse \\ Prof. Dr. K.-F. Becker}

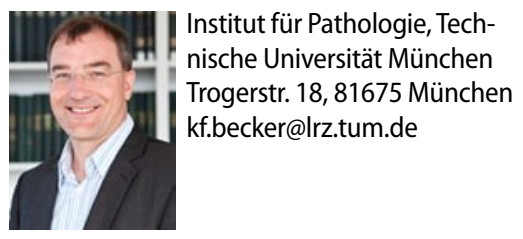

Danksagung. Unsere Arbeiten werden durch die EU (,SPIDIA“) und das BMBF (Förderkennzeichen $\mathrm{Nr}$. 01GR0805, „FFPE-Gewebe ") gefördert.

Interessenkonflikt. Der korrespondierende Autor weist auf folgende Beziehung hin: KFB ist als Erfinder für ein Patent zur Proteinextraktion aus FFPE-Geweben genannt.

\section{Literatur}

1. Addis MF, Tanca A, Pagnozzi D et al (2009) Generation of high-quality protein extracts from formalin-fixed, paraffin-embedded tissues. Proteomics 9:3815-3823
2. Ahram M, Flaig MJ, Gillespie JW et al (2003) Evaluation of ethanol-fixed, paraffin-embedded tissues for proteomic applications. Proteomics 3:413-421

3. Becker KF, Metzger V, Hipp S, Hofler H (2006) Clinical proteomics: new trends for protein microarrays. Curr Med Chem 13:1831-1837

4. Becker KF, Schott C, Hipp S (2007) Quantitative protein analysis from formalin-fixed tissues: implications for translational clinical research and nanoscale molecular diagnosis. J Pathol 211:370-378

5. Becker KF, Schott C, Becker I, Höfler H (2008) Guided protein extraction from formalin-fixed tissues for quantitative multiplex analysis avoids detrimental effects of histological stains. Proteomics Clin Appl 2:737-743

6. Berg D, Hipp S, Malinowsky K et al (2010) Molecular profiling of signalling pathways in formalinfixed and paraffin-embedded cancer tissues. Eur J Cancer 46:47-55

7. Chung JY, Lee SJ, Kris Y et al (2008) A well-based reverse-phase protein array applicable to extracts from formalin-fixed paraffin-embedded tissue. Proteomics Clin Appl 2:1539-1547

8. Djidja MC, Claude E, Snel MF et al (2009) MALDIion mobility separation-mass spectrometry imaging of glucose-regulated protein $78 \mathrm{kDa}$ (Grp78) in human formalin-fixed, paraffin-embedded pancreatic adenocarcinoma tissue sections. J Proteome Res 8:4876-4884

9. Djidja MC, Francese S, Loadman PM et al (2009) Detergent addition to tryptic digests and ion mobility separation prior to MS/MS improves peptide yield and protein identification for in situ proteomic investigation of frozen and formalin-fixed paraffin-embedded adenocarcinoma tissue sections. Proteomics 9:2750-2763

10. Espina V, Mehta Al, Winters ME et al (2003) Protein microarrays: molecular profiling technologies for clinical specimens. Proteomics 3:2091-2100

11. Espina V, Edmiston KH, Heiby M et al (2008) A portrait of tissue phosphoprotein stability in the clinical tissue procurement process. Mol Cell Proteomics 7:1998-2018

12. Feinberg JG (1961) A,microspot' test for antigens and antibodies. Nature 192:985-986

13. Gillespie JW, Best CJ, Bichsel VE et al (2002) Evaluation of non-formalin tissue fixation for molecular profiling studies. Am J Pathol 160:449-457

14. Groseclose MR, Massion PP, Chaurand P, Caprioli RM (2008) High-throughput proteomic analysis of formalin-fixed paraffin-embedded tissue microarrays using MALDI imaging mass spectrometry. Proteomics 8:3715-3724

15. Gulmann C, Sheehan KM, Kay EW et al (2006) Array-based proteomics: mapping of protein circuitries for diagnostics, prognostics, and therapy guidance in cancer. J Pathol 208:595-606

16. Hartmann A (2006) Role of predictive pathology in oncology - examples of new therapies targeting EGFR. Verh Dtsch Ges Pathol 90:128-135

17. Hewitt SM, Lewis FA, Cao Y (2008) Tissue handling and specimen preparation in surgical pathology: issues concerning the recovery of nucleic acids from formalin-fixed, paraffin-embedded tissue. Arch Pathol Lab Med 132:1929-1935

18. Ikeda K, Monden T, Kanoh T (1998) Extraction and analysis of diagnostically useful proteins from formalin-fixed, paraffin-embedded tissue sections. J Histochem Cytochem 46:397-403

19. Lemaire R, Desmons A, Tabet JC (2007) Direct analysis and MALDI imaging of formalin-fixed, paraffin-embedded tissue sections. J Proteome Res 6:1295-1305
20. Liotta LA, Espina V, Mehta Al (2003) Protein microarrays: meeting analytical challenges for clinical applications. Cancer Cell 3:317-325

21. Nirmalan NJ, Harnden P, Selby PJ, Banks RE (2009) Development and validation of a novel protein extraction methodology for quantitation of protein expression in formalin-fixed paraffin-embedded tissues using western blotting. J Pathol 217:497506

22. Paweletz CP, Charboneau L, Bichsel VE et al (2001) Reverse phase protein microarrays which capture disease progression show activation of pro-survival pathways at the cancer invasion front. Oncogene 20:1981-1989

23. Petricoin EF 3rd, Bichsel VE, Calvert VS et al (2005) Mapping molecular networks using proteomics: a vision for patient-tailored combination therapy. J Clin Oncol 23:3614-3621

24. Ronci M, Bonanno E, Colantoni A et al (2008) Protein unlocking procedures of formalin-fixed paraffin-embedded tissues: application to MALDI-TOF imaging MS investigations. Proteomics 8:37023714

25. Seeley EH, Caprioli RM (2008) Molecular imaging of proteins in tissues by mass spectrometry. Proc Natl Acad Sci U S A 105:18126-18131

26. Shi SR, Liu C, Balgley BM et al (2006) Protein extraction from formalin-fixed, paraffin-embedded tissue sections: quality evaluation by mass spectrometry. J Histochem Cytochem 54:739-743

27. Stauber J, Lemaire R, Franck J et al (2008) MALDI imaging of formalin-fixed paraffin-embedded tissues: application to model animals of Parkinson disease for biomarker hunting. J Proteome Res 7:969-978

28. Stauber J, MacAleese L, Franck J et al (2010) On-tissue protein identification and imaging by MALDIion mobility mass spectrometry. J Am Soc Mass Spectrom 21:338-347

29. Tibes R, Qiu Y, Lu Y et al (2006) Reverse phase protein array: validation of a novel proteomic technology and utility for analysis of primary leukemia specimens and hematopoietic stem cells. Mol Cancer Ther 5:2512-2521

30. Viertler C, Zatloukal K (2008) Biobanking and Biomolecular Resources Research Infrastructure (BBMRI). Implications for pathology. Pathologe 29:210-213

31. Walch A, Rauser S, Deininger SO, Höfler H (2008) MALDI imaging mass spectrometry for direct tissue analysis: a new frontier for molecular histology. Histochem Cell Biol 130:421-434

32. Wulfkuhle JD, Edmiston KH, Liotta LA, Petricoin EF 3rd (2006) Technology insight: pharmacoproteomics for cancer - promises of patient-tailored medicine using protein microarrays. Nat Clin Pract Oncol 3:256-268 